\title{
UWA-NAV - Energy Efficient Error Control Scheme for Underwater Acoustic Sensor Network*
}

\author{
Soo-Young Shin and Soo-Hyun Park ${ }^{* *}$ \\ Graduate School of BIT, Kookmin University \\ \{sy-shin, shpark21\}@kookmin.ac.kr
}

\begin{abstract}
Since there are various disturbing factors such as long propagation delay and high error rate in UW-ASN, an error control mechanism is required to improve the system performance efficiently. In this paper, the carrier sensing technique, which detects whether the public media access is already occupied or not and evade the collision, is used as an error control mechanism. A method of monitoring the energy level of wireless frequency in physical layers and a method of setting-up NAV (Network Allocation Vector) using the frame transmission period were modified and re-defined for underwater environment. Through these works, a more efficient UWA-NAV technique was able to be proposed and the performance of the proposed technique was evaluated and compared with the case without UWA-NAV.
\end{abstract}

Keywords: UW-ASN, NAV, Congestion Control, Error Control, Energy Aware.

\section{Introduction}

The connection technology of IEEE 802.11x has adopted CSMA/CA (Carrier Sensing Multiple Access / Collision Avoidance) technique. CSMA/CA uses carrier sensing technique for identifying whether the transmission media is used by other nodes or not. There are two methods of carrier sensing. One method is monitoring energy level of wireless frequency at physical layer. Another method specifies NAV (Network Allocation Vector) using frame transmission period virtually. It is called virtual carrier sensing. NAV means remaining time of current transmission session. Through $\mathrm{NAV}$, it can be detected whether other nodes are transmitting or not. In other word, if NAV is set up then it means that the transmission route is occupied by some nodes[1][2].

The proposed concept of UWA-NAV in underwater sensor network is different from that of wireless communication. The proposed method occupies the transmission

\footnotetext{
* This research was supported by the MIC (Ministry of Information and Communication), Korea, under the 2007 ITRC (Information Technology Research Center) support program supervised by the IITA (Institute of Information Technology Assessment).

** Corresponding author.
} 
time using NAV and Master measures the network congestion. And then according to the measured value, deadlock state is defined to stop data transmission for a certain period and prevent system resource and energy consumption from wasting. In addition, the purpose of UWA-NAV is diverse. It can be used for Master's data transmission, network reconfiguration, new nodes' joining with network and etc. NAV is used to prevent system resource, such as energy and bandwidth, from wasting and stops all data transmission for a certain period. In this paper, by using UW-NAV technique, a method, which is adaptive to rapidly fluctuating transmission failure rate, was proposed for reducing energy consumption.

The structure of this paper is as follows. In Chapter 2, NAV technique of IEEE $802.11 \mathrm{x}$ is briefly reviewed. In Chapter 3, the proposed UWA-NAV technique is described in details. In Chapter 4, the performance of the proposed method is analyzed. Lastly conclusions are in Chapter 5.

\section{NAV in IEEE 802.11x [1][2]}

The Network Allocation Vector (NAV) is a method to avoid collisions in a shared transmission medium.

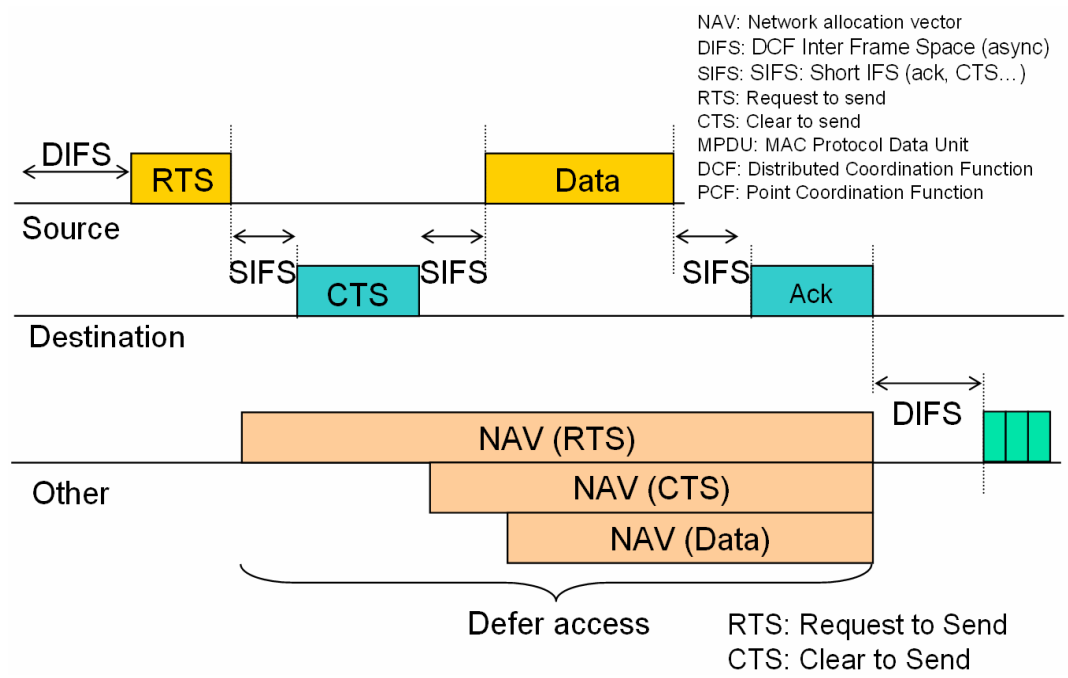

(A. Leon-Garcia, I. Widjaja, Communication Networks, Instructor's Slide Set)

Fig. 1. NAV Operation

Each station wishing to transmit through the shared medium performs a RTS with a NAV that indicates the time required to complete the whished transmission. If no collision is detected and after the acknowledge published by the receiving station in a 
CTS packet, every connected station consider the shared medium allocated to the sending station during the time specified in the NAV. This technique is used within IEEE 802.11 (Wi-Fi) and IEEE 802.16 (WiMax) networks [3].

\section{NAV and Congestion Control in Underwater Communication}

\subsection{Underwater Acoustic Sensor Network (UW-ASN) MAC Mechanism}

In this section, several presuppositions with brief descriptions of MAC design were mentioned. (Author has been studying MAC systems for UW-ASN). Firstly, the proposed MAC system is for monitoring sea environment with 2D topology of cluster-type. No moving object (AUV) is considered and TDMA (Time Division Multiple Access) is adopted for communication between clusters. Nodes will transmit data periodically according to the pre-defined schedule. The system has 5 steps for its communication
1) Network Initiation and configuration $(\mathrm{CH}+\mathrm{SNs})$
2) Broadcast to all member node with $\mathrm{Acks}(\mathrm{CH})$
3) Data transmission ( SNs)
4) Repeat 2) 3) step
5) Network reconfiguration $(\mathrm{CH}+\mathrm{SNs})$

More detailed explanation and system spec can be found in [4] and [5].

\subsection{UWA-NAV}

In this section, a new definition of UWA-NAV and its procedure were explained. The necessity of UWA-NAV depends on the sea environment. Since Acoustic is used for communication, there are many sources of artificial and natural noise such as low frequency sounds generated by ships and machines, high frequency sounds generated by dolphins. The sources and their effects are Seismo-acoustic noise, Shipping noise, Bio-acoustic noise, Wind and rain noise, Depth dependence, Directionality, Arctic ambient noise, Acoustic daylight and etc. For last 3 decades, many researchers have conducted various tests and modeling works to make better noise model[6]. The latest version of numerical noise model (RANDI III - The research ambient-noise directionality III) was developed for application to shallow-water and coastal areas in the low-to-mid frequency range $(\sim 10-300 \mathrm{~Hz})[6]$. The receiver can be either a horizontal or a vertical line array (Breeding, 1993). However, no unified model considering various sea environments was proposed yet and still it remains as a challenging research area [6].

Firstly, UWA-NAV is handled by $\mathrm{CH}$. $\mathrm{CH}$ is a scheduler for unit network and controls TDMA scheduling, various guard band settings, Ack transmission, Power saving mode controls and etc. It also controls the waste of the energy resource using UWA-NAV flag. When the transmission rate of the network is remarkably decreased or error rate is rapidly increased, $\mathrm{CH}$ can calculate the trade-off relationship between 
the transmission efficiency and the energy consumption rate by measuring SNR or referring LQI information. In case of conducting non transmission mode according to a certain policy based on pre-defined threshold value, devices can prevent the waste of network resource and energy consumption resulted from significantly increased transmission failure rate by maintaining power saving mode and stopping data transmission during a certain period. However, as for the number of packets which are succeeded in transmission, the conventional method can process more packets than the proposed method. Based on the fact that if error rate gets higher than a certain level then the success rate of packet transmission increases rapidly, the proposed method can be a scheme preventing serious waste of network resource [4].

\subsection{UWA-NAV Flag}

Figure 2 shows an example of frame format for values of UWA-NAV included and transmitted with Control (Beacon) Packets.

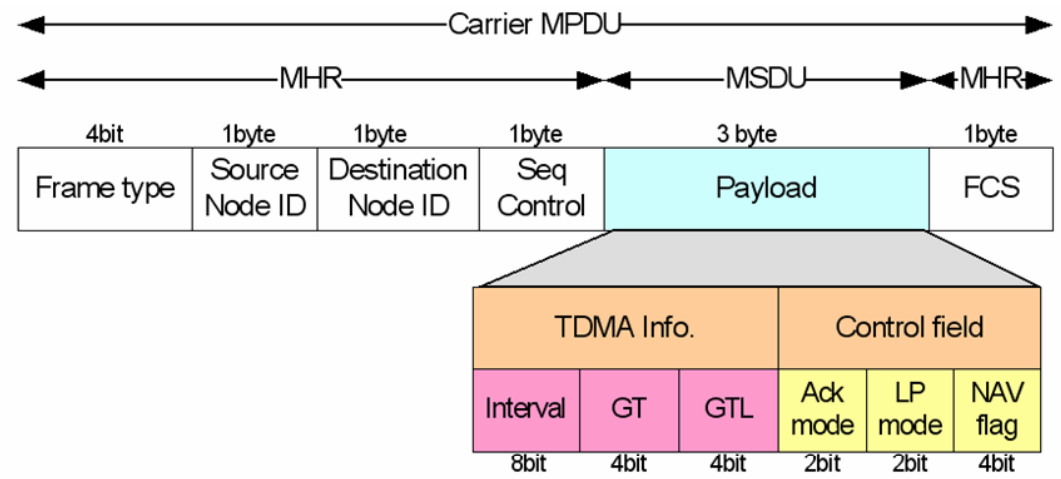

Fig. 2. An Example of Control (Beacon) Frame with NAV flag

Table 1. An Example of NAV flag

\begin{tabular}{|c|c|c|c|}
\hline \multirow{9}{*}{$\begin{array}{c}\text { NAV } \\
\text { Flag } \\
\text { (4bits) }\end{array}$} & \multirow{8}{*}{$\begin{array}{l}\text { Non transmission } \\
\text { mode }\end{array}$} & 0000 & 1 Level Deadlock state for error control \\
\hline & & 0001 & 2 Level Deadlock state for error control \\
\hline & & 0010 & 3 Level Deadlock state for error control \\
\hline & & 0011 & 4 Level Deadlock state for error control \\
\hline & & 0100 & 5 Level Deadlock state for error control \\
\hline & & 0101 & 6 Level Deadlock state for error control \\
\hline & & 0110 & 7 Level Deadlock state for error control \\
\hline & & 0111 & 8 Level Deadlock state for error control \\
\hline & $\begin{array}{c}\text { Transmission } \\
\text { mode }\end{array}$ & $\begin{array}{c}1000 \sim \\
1111\end{array}$ & Reserved \\
\hline
\end{tabular}


NAV flag of 4 bits included in Control field can operates in Non transmission mode as shown in the table below. 8 steps of adaptive error controls are possible and NAV duration time is also controllable according to its error rate. Threshold value can be adjusted by a system manager and the required field among 1 8 steps can be specified taking various environmental condition into consideration.

\subsection{NAV Flag Generation Procedure}

For the calculation of NAV flag, the measurement of SNR (or LQI) value and delay time of network has to be done. The delay time can be measured together with the propagation delay by using the information of time stamp of network initiation and data transmission. SNR and LQI are values for measurement of error rate which has been typically used in sensor network system. At this time, since a policy table of user defined UWA-NAV already exists, the final NAV flag can be set-up.

Figure 3 shows the conceptual diagram of NAV flag generation procedure.

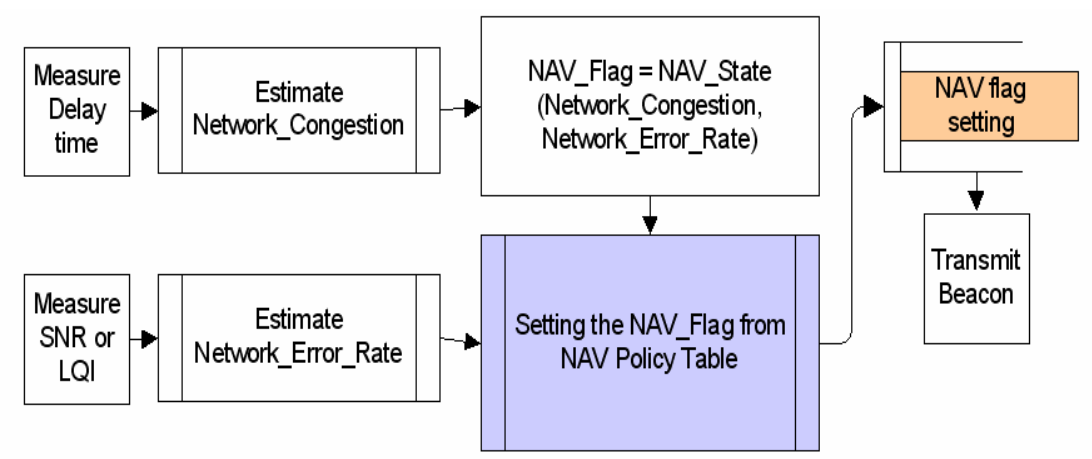

Fig. 3. NAV flag Generation Procedure (CH side)

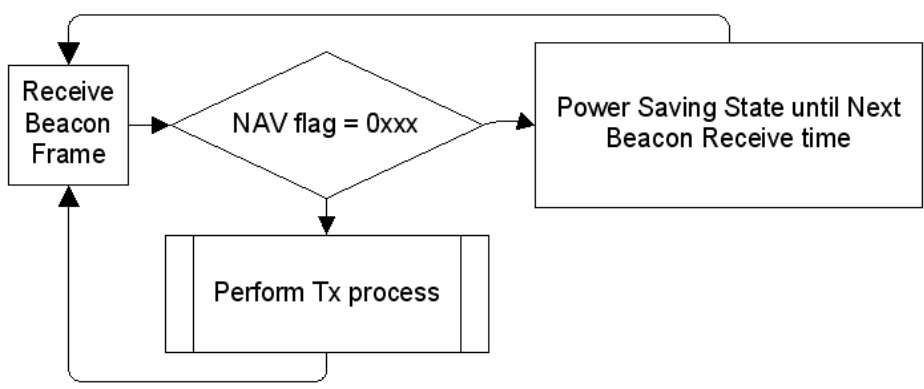

Fig. 4. UWA-NAV Transmission Procedure (sensor node side) 
After $\mathrm{CH}$ transmits NAV flag which is included in Beacon frame, the sensor node, which is located in the receiving cluster, stops transmitting for a certain time according to the types of NAV policy table and maintains power saving mode until the next receiving of Beacon frame. And the sensor node repeats the same procedure until the newly received NAV flag of Beacon frame is changed and the network communication is re-opened again. Figure 4 shows the brief procedure seen from the sensor node side.

\section{Simulation Result}

In this paper, a moving ship is assumed as a constant noise source and a periodic data acquisition in shallow water by the network is assumed. This system presupposes the situation of receiving sea environment monitoring data from sensor nodes and transmitting the data to BS (surface). Transmission failure and the waste of network resource caused by very high error rate are compared from the point of energy consumption. Table 2 shows the parameters for the performance comparison.

Table 2. Simulation parameters

\begin{tabular}{|l|l|}
\hline \multicolumn{1}{|c|}{ Parameters } & \multicolumn{1}{c|}{ Values } \\
\hline Simulation time & $1,000 \mathrm{Sec}$ \\
\hline Data transmission & 100 packets $/ \mathrm{sec}$ \\
\hline Noise duration time & $50 \sim 800 \mathrm{Sec}$ \\
\hline Error reduction rate & $30 \%$ \\
\hline Fluctuation of error rate & $50 \%$ \\
\hline Noise Source & $\mathrm{A}$ vessel \\
\hline Energy exhaustion unit (EEU) & 1 (per 100 transmit +100 receive) \\
\hline Default error rate & $10^{-1} \sim 2^{*} 10^{-1}$ \\
\hline
\end{tabular}

10 steps of the noise duration times are generated and the performance is calculated and analyzed. Figure 5 shows the success rate of transmission-tried packets (Figure 5(a)), transmission rate of overall network (Figure 5(b)), energy consumption of the network (Figure 5(c)) and the energy efficiency (Figure 5(d)).

In case that the system processing rate decreases rapidly by constant occurrence of transmission error and the transmission is prevented by the value of UWA-NAV, the transmission efficiency is improved as the noise duration time increases comparing with normal case. It is because the number of total $\mathrm{Tx} / \mathrm{Rx}$ done packets increased although the success rate is small. 


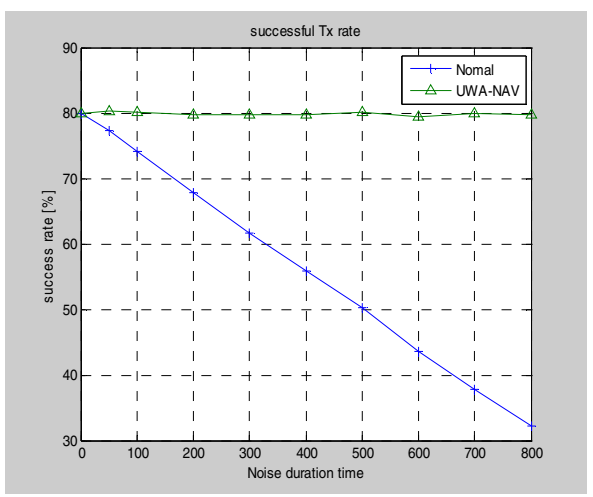

(a) Successful Tx rate

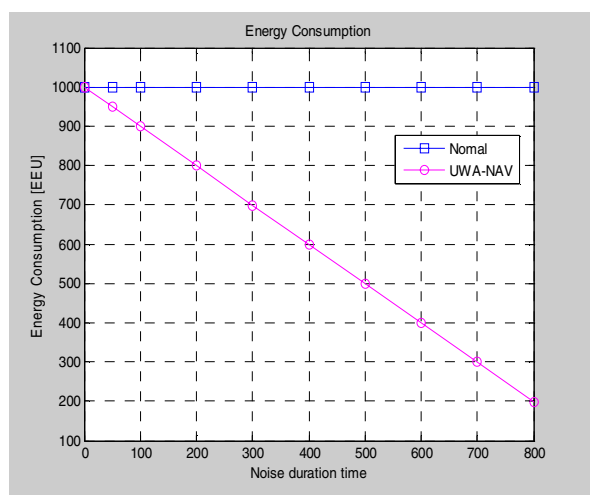

(c) Energy consumption

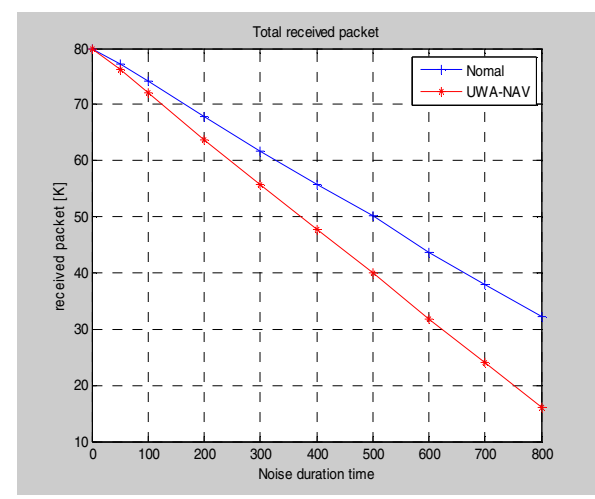

(b) Total Tx/Rx done packet

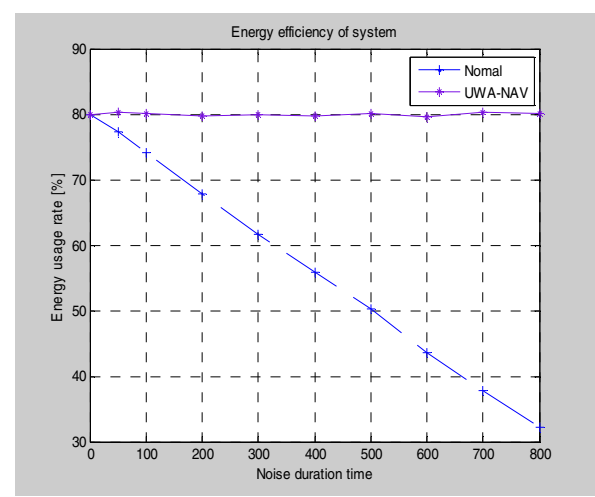

(d) Energy efficiency of system

Fig. 5. Simulation results

\section{Conclusion}

In this paper, a method for reducing the waste of system resources and energy consumption was proposed. The proposed method occupies transmission period by using UWA-NAV and the network congestion is measured by Master. Based on the measured values, Deadlock state is set up to stop data transmission to improve system's resource usage and its energy efficiency. Simulations were conducted to verify the performance of the proposed method which is adaptive to severe fluctuation of the transmission failure rate caused by constant noise source in underwater network environment.

UWA-NAV is not adequate to real time data transmission which has a limitation of transmission times. It is because that, during noise generating period, time delay is caused and there can be data without change of transmission trial in case of not enough carrying queue in the system. It is expected that these problems can be solved by aggregation technique and fitter. Further study for ensuring data transmission reliability and transmission mode of UWA-NAV is planned. 


\section{References}

[1] Wireless LAN medium access control (MAC) and physical layer (PHY) specifications: higher speed physical layer (PHY) extension in the $2.4 \mathrm{GHz}$ band, IEEE Std $802.11 \mathrm{~b}$ (April 1999)

[2] IEEE Standard 802.11e-2005 (Amendment to IEEE Std 802.11, 1999 Edition (Reaff 2003) (2005)

[3] Liu, H.-H., Wu, J.-L.C., Chen, W.-Y.: New frame-based network allocation vector for 802.11b multirate wireless LANs, Communications. IEE Proceedings 149(3), 147-151 (2002)

[4] Shin, S.Y.: Smart Blocking Media Access Control Mechanism for UW-ASN, Thesis for the Degree of Ph. D, Kookmin Univ. (January 2007)

[5] Shin, S.Y., Choi, J.K., Park, S.H.: Transmission Environment for Underwater Acoustic Communication. In: ITPA 2007 (September 2007) (will be published)

[6] Paul, C.: Underwater Acoustic Modeling and Simulation. Spon Press, London (2003)

\section{Appendix}

We classified the transmission environment into 9 cases. Figure 6 shows the simulation results of noise duration time ranging from 50 to 800 seconds. The left figure shows the real time transmission success rate in percentage and the right one represents the energy consumption value (EEU).
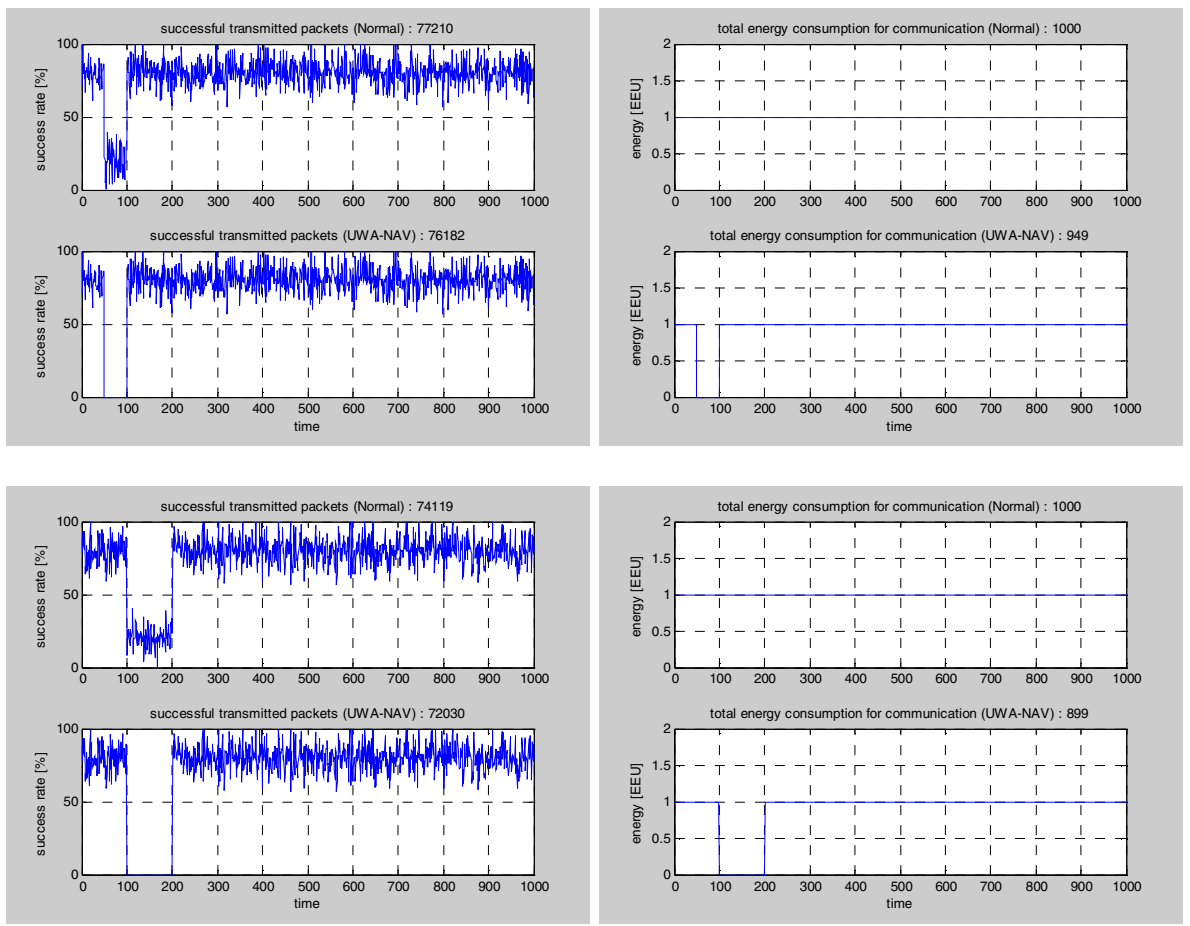

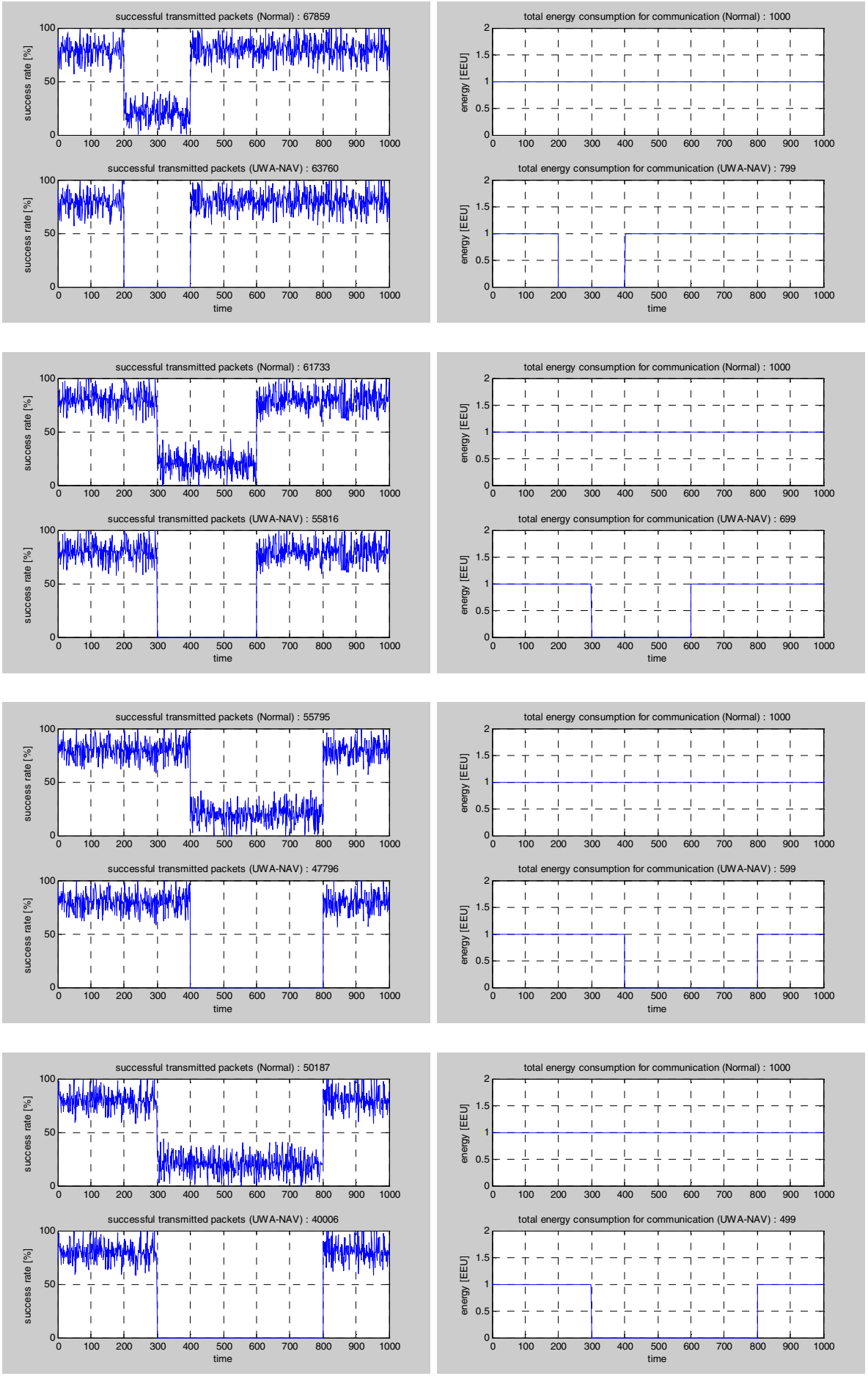

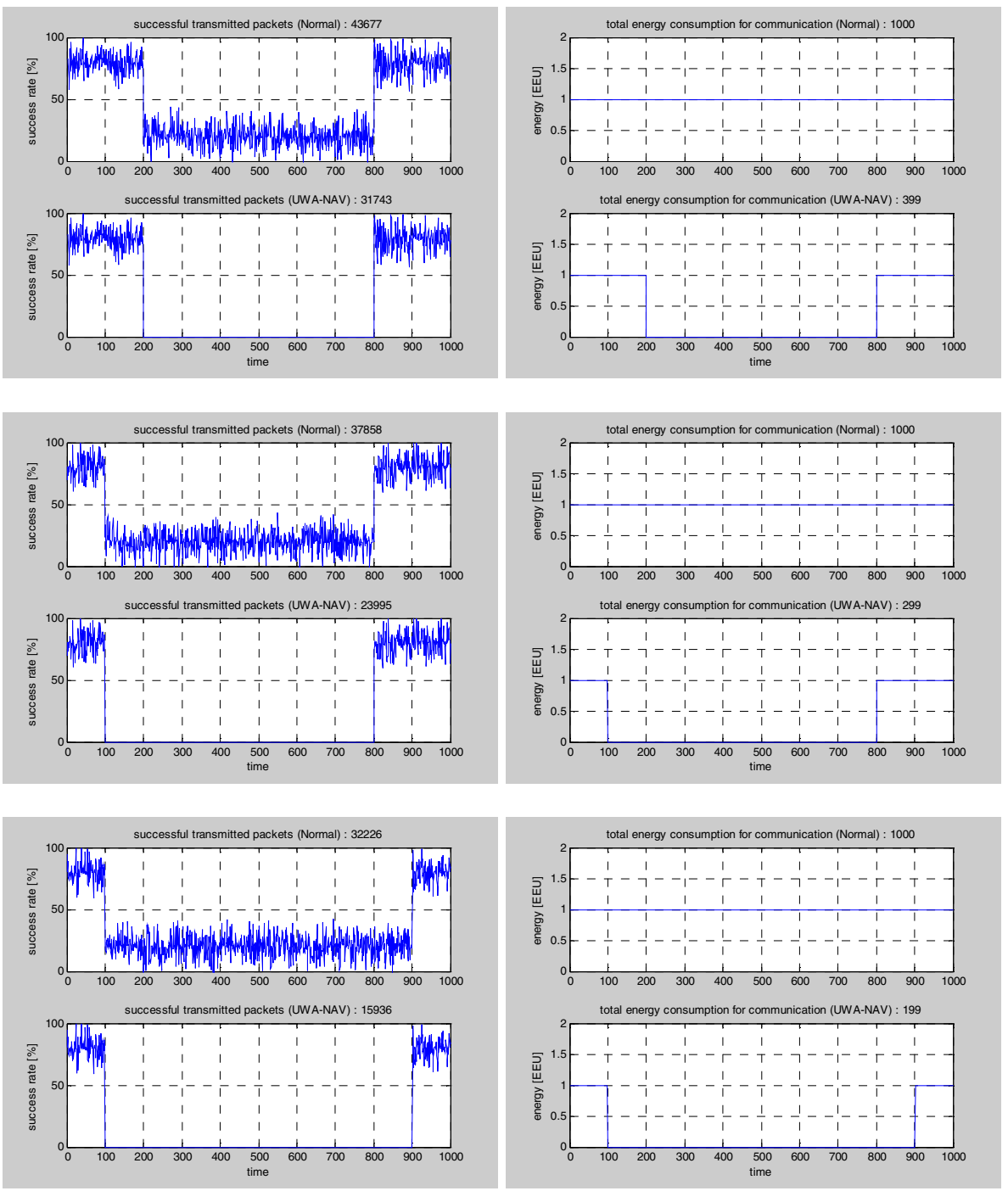

Fig. 6. Tx/Rx packets and Energy consumption (Noise duration time: 50 800 sec) 